\title{
La Responsabilidad Social Empresarial: aplicándola para tener un mundo mejor
}

\author{
Corporate Social Responsibility: applying for a better world
}

\author{
Fidel Arturo LÓPEZ EGUIZÁBAL ${ }^{1}$ \\ Universidad Francisco Gavidia (San Salvador) \\ flopez@ufg.edu.sv
}

Recibido: 13 de junio del 2014 Aceptado: 07 de septiembre 2014

\begin{abstract}
Resumen
Las empresas ya sean bancos, iglesias, colegios, universidades, gremiales empresariales, Ong's y otras instituciones, buscan constantemente posicionarse en la mente de los consumidores, de vender sus productos y servicios a través de diferentes estrategias comunicativas.

Las empresas y universidades, puntos clave de este documento, son los que deben unirse con el gobierno para poder hacer un holding estratégico y trabajar en conjunto para dejar un mejor país. Lo anterior lo encontramos en una leyenda de la Asociación Scouts "Intentad dejar este mundo un poco mejor de cómo os lo encontrasteis". Robert Baden Powell.

Los empresarios en especial son los principales entes que tiene que involucrar más a programas de responsabilidad social empresarial. Los empleados, los clientes, las comunidades y demás personas que se involucran con la empresa, tendrán mejor calidad de vida.

La RSE según Peter Drucker "Es una ética corporativa para abordar la forma en que la empresa trata a sus constituyentes internos y externos y el mundo en que ellos viven."

Que no se tome nada más como una actividad para evadir impuestos o para mejorar la imagen. Que la RSE sea ejecutada desde las políticas empresariales. Todos los seres humanos deseamos vivir mejor, de trabajar en ambientes con un clima y cultura organizacional idóneos.
\end{abstract}

\footnotetext{
${ }^{1}$ Catedrático Universidad Francisco Gavidia UFG y docente investigador en el Instituto Ciencia Tecnología e Innovación de la UFG. Maestría en Profesionalización de la Docencia Superior Universidad de El Salvador y Licenciado en Relaciones Públicas y Comunicaciones Universidad Tecnológica de El Salvador.
} 
Una de las estrategias más empleadas en relaciones públicas es la Responsabilidad Social Corporativa o Responsabilidad Social Empresarial RSE. Sin embargo que no se tome a la RSE como una herramienta filantrópica o una forma de buscar protagonismo. En países desarrollados, a las empresas las tienen bien evaluadas con respecto a programas de RSE. En el país, hay instituciones, personas y colaboradores que se involucran con modelos justos de RSE a una sana aplicación.

\section{Abstract}

Companies such as banks, churches, schools, universities, business associations, NGOs and other institutions are constantly seeking to position themselves in the minds of consumers, to sell their products and services through different communication strategies.

Companies and universities, key points of this document, are to be joined with the government to make a strategic holding and work together to leave a better country. This is found in a legend of the Scouts Association "Try to leave this world a little better than how you found it". Robert Baden Powell.

Entrepreneurs in particular are the main entities that must involve more corporate social responsibility programs. Employees, customers, communities and other people involved with the company, will have better quality of life.

CSR according to Peter Drucker "It is a corporate ethics to address how the company treats its internal and external constituents and the world in which they live."

That does not take anything more as an activity to evade taxes or to improve the image. CSR to be executed from business policies. All human beings want to live better, to work in environments with suitable organizational climate and culture.

One of the strategies used in public relations is the Corporate Social Responsibility or CSR Corporate Social Responsibility. But do not take CSR as philanthropic tool or a way to search prominence. In developed countries, the companies have well evaluated for CSR programs. In the country, there are institutions, people and partners who engage with CSR models just to sound application.

Palabras clave: Responsabilidad; ambiental; relaciones públicas; social; empresario; corporativo; valores.

Keywords: Accountability; environmental; public relations; social; business; corporate; securities. 


\section{1. ¿Cuáles son las empresas salvadoreñas que aplican RSE?}

Toda empresa sea pequeña o grande, tiene que incorporar programas de RSE, no es una obligación, empero los resultados, aunque se invierta dinero y recursos, hacen tener mejores empleados, comunidades beneficiadas y una sociedad con mejor calidad de vida.

Empresas del gobierno, empresa privada, las del sector turismo y otras, pueden aplicar programas de RSE desde recolección de desechos tóxicos, limpieza de playas, ciudades, brindar becas, donar equipo como computadoras, colaborar con el personal en catástrofes, etc. Algunas empresas ni si quiera hacen un mínimo esfuerzo de devolverle a la sociedad una retribución por haberse desarrollado. No invierten debido a que piensan que es un costo extra.

Las empresas salvadoreñas que aplican el modelo estratégico de RSE están:

Banco Agrícola (Banco colombiano)

Banco Davivienda (El Salvador)

Industrias La Constancia

Microsoft de El Salvador

Grupo CASSA (Compañía Azucarera Salvadoreña)

Hotel Crowne Plaza (El Salvador)

Holiday Inn (El Salvador)

Kimberly Clark (El Salvador)

Grupo Calvo

PYMES como Salvanoni y Arte Comasagua.

Universidades, iglesias, gremiales, etcétera.

En las aulas universitarias se enseña que al aplicar RSE las empresas tendrán mejores resultados hacia la comunidad, públicos internos y un mejor ambiente laboral, que es de los puntos más importantes.

Donaciones millonarias a fundaciones y ONG, formación de clubes deportivos para niños de escasos recursos y entrega de dotaciones a escuelas de barrios marginales son algunas actividades que pueden ser parte de un plan de responsabilidad social empresarial, RSE, pero no son lo único. 
Es importante recargar que existen empresas que no son salvadoreñas, ejemplo es el Grupo Calvo, el cual tiene programas de RSE destacados.

“El Grupo Calvo entró en El Salvador en el año 2003, cuando inauguró la fábrica en la ciudad de La Unión. La Cámara de Comercio ha destacado varios criterios por los que distingue a Calvo con La Palma de Oro, como el importante impulso económico y social que ha aportado la compañía a las comunidades del departamento de La Unión y a otros sectores de la población, por su calidad humana y por sus proyectos de responsabilidad social".

El grupo Calvo ayuda a la alimentación de sus empleados, ha ayudado a la alfabetización de la población, campañas de salud visual y vacunación.

Sin embargo, uno de los más importantes es: "De todos estos programas, uno de los más importantes para el Grupo es el de la reinserción laboral de ex pandilleros salvadoreños. Esta iniciativa cuenta con el reconocimiento de la sociedad de La Unión y con el respaldo del Gobierno de El Salvador".

\section{Las relaciones públicas y aplicabilidad de la Responsabilidad Social Empresarial en las empresas}

En la experiencia como relacionista público y catedrático, evidencio que las empresas buscan ser reconocidas, de salir del anonimato y, a través de la RSE y con la variedad de actividades, logran posicionarse en rankings nacionales y ser reconocidas por la colaboración empresarial hacia la sociedad. Las empresas aplican programas de relaciones públicas con el objetivo de dar a conocer a la empresa.

Responsabilidad Social Empresarial (RSE): es un imperativo estratégico que le permite a la empresa incorporar políticas y prácticas en beneficio de los accionistas, los colaboradores, la comunidad, el medio ambiente y toda su cadena de valor; a través de la alineación de su gestión con principios éticos y transparencia, convirtiéndola en un agente competitivo que contribuye al desarrollo económico y social.

Sostenibilidad: Son aquellas actividades y procesos que son capaces de desarrollarse por sus propios recursos y medios sin comprometer recursos a futuro.

Un aspecto importante en la responsabilidad social corporativa es la filantropía corporativa la cual hace que las empresas se identifiquen más con los diferentes públicos y esto hace que mejore la imagen. Entre los beneficios que ayudan al aplicar la filantropía corporativa están: 
Reputación fortalecida y reconocimiento de marca.

Mayores oportunidades en los medios de comunicación.

Mejore relaciones con la comunidad y gubernamentales.

Facilidad para reclutar y mantener a los empleados.

Mejor marketing y relaciones públicas.

Acceso a la investigación y desarrollo.

Mayor rentabilidad empresarial.

Las relaciones públicas actúan como estrategia cuando hay donaciones o campañas. En investigación de tesis de la Universidad Centroamericana José Simeón Cañas, se encuentra datos relacionados a la RSE, a saber:

De acuerdo con Muñoz, Guardado y Lazo (2005, p. 14) el campo de acción de las Relaciones Públicas es el siguiente: salvaguardar la imagen de la empresa, mantener buenas relaciones con los públicos, ser portavoz de la empresa, organizar eventos, mejora de conflicto y programas de RSE.

Estos programas hacen referencia al conjunto de obligaciones y compromisos, legales y éticos, que se derivan de los impactos que la actividad de las organizaciones producen en el ámbito social, laboral, medioambiental y de los derechos humanos.

El beneficio que conlleva actuar en el campo de acción de los programas de la RSE es que eleva el nivel de credibilidad con la comunidad donde las compañías contribuyen a mejorar el ambiente y el entorno en el que operan correspondiendo a las necesidades de la sociedad, haciendo lo que es ético, justo y acorde a la ley siendo un buen ciudadano empresarial.

Estas campañas de RSE tienen ciertas características:

Son muy diversas en cuanto a los temas o necesidades comunitarias.

Son menos sistemáticas y organizadas que las campañas comerciales.

Son realizadas por una variedad de fuentes que patrocinan el esfuerzo persuasivo (gobierno, bancos, clubes, organizaciones altruistas, etc.)

Hay menos consideración entre los equipos de trabajo y más rotación de personal no profesional. 
La extensión es variable, limitada a un grupo, ciudad, región o país en forma independiente $\mathrm{o}$ en unión con otros grupos a nivel mundial.

Las empresas salvadoreñas en su mayoría de tamaño grande utilizan mucho esta estrategia para aumentar su imagen, reciclan sus desechos tóxicos, reciclan papel, ayudan a las comunidades, incorporan campañas en pro del medio ambiente, brindan becas, estimulan a los empleados brindándoles capacitaciones, estabilidad laboral, educación, clínicas empresariales, etc.

Según FUNDEMAS, los beneficios que reciben las empresas al aplicar la RSE son:

Disminución de conflictos

Acceso a capital

Valorización de la imagen institucional y de su marca

Mayor lealtad del consumidor

Mayor capacidad de reclutar y mantener sus talentos

Sustentabilidad del negocio a largo plazo

Acceso a mercados

En los anteriores aspectos la valorización de la imagen institucional y de su marca es lo que nos compete y es lo que las empresas buscan al aplicar la Responsabilidad Social Empresarial.

Según publicación en un periódico: "En el caso de Microsoft, Giancarlo Orsenigo, gerente de mercadeo en el país, los programas y la concepción de la institución en este tema han beneficiado al reducir la rotación de personal. Los empresarios coinciden en que a largo y a corto plazo, cumplir con su responsabilidad social en la comunidad es un "ganar-ganar".

Otro caso es el de apoyar el medio ambiente, recordemos que en el país a pesar de tener un Ministro del Medio Ambiente, las leyes no se cumplen en el caso de proteger al medio ambiente.

Banco Agrícola es otra de las empresas que cree firmemente en la necesidad de ayudar a la sociedad, específicamente en educación y el medio ambiente.

Joaquín Rivas, gerente de comunicaciones de Banco Agrícola, asegura que desde que nació la institución se han realizado diversas acciones orientadas a contribuir al desarrollo del país. 
En 1994, se impulsó de lleno la implementación de prácticas de RSE al lanzar el programa Un Rincón Mágico, con el cual se busca fomentar la cultura y difundir una imagen positiva de El Salvador. Este incluye la edición de un libro con temas salvadoreños, el cual es entregado a consulados, diplomáticos y sectores que puedan promover la imagen del país en el exterior. Se han editado 14 libros con esa temática y, este año, el libro estará dedicado a las aves de El Salvador.

En entrevista realizada a la Licda. Mariela Franco Jefa de Responsabilidad Social del Banco Agrícola, manifestó:

¿En qué ayuda la RSE a las empresas?

"La RSE ayuda a la reputación de las empresas, en el banco contamos con cinco ejes de acción o actividades principales que realizan en aspectos de una cultura empresarial con responsabilidad social"

¿Qué actividades hacen en el área de Responsabilidad Social Empresarial?

"Entre las actividades que el banco realizan están: Programa Manos Unidas por El Salvador, Programa de Apoyo a la Ecología, Programa de Fomento Cultural, Programa de Educación Financiera y otros programas como El banco de los niños".

¿Han ganado premios por aplicar la RSE?

Aplicar la RSE implica no solo realizar actividades para ganar premios, es un compromiso que se tiene con la sociedad de contribuir al desarrollo, el Banco Agrícola es siempre premiado en el Ranking de imagen de la Universidad Dr. José Matías Delgado.

¿Tienen ustedes departamento de RRPP para aplicar mejor la RSE?

"No tenemos departamento de RRPP, la gerencia de comunicaciones se encarga de todo las actividades, nada menos ayer nos reunimos las Gerencia de gestión humana y se dieron a conocer la labor que desarrolla el banco en este trimestre, además tendremos la inauguración de una comunidad que se llama Esmeralda, el banco ha construido más de veinte casas para personas de escasos recursos y la inauguración será en marzo del 2012.

¿Cómo realizaron esa actividad?

"La alcaldía donó el terreno y luego el banco las construyó y la equipó, las familias se pasarán a vivir en ellas el 16 de marzo previo a la inauguración y entrega de casas".

Por lo tanto se evidencia que en el Banco Agrícola las actividades de RSC ayudan a mejorar su reputación y por ende a incrementar los clientes. 
Otro caso ejemplar es el que realizó Industrias La Constancia para contribuir al medio ambiente, la empresa ayuda al medioambiente en reciclar las aguas que salen del área de producción y luego son tratadas en posos para luego sean depositadas a los ríos sin contaminación alguna.

A esta empresa tuve el gusto de conocerla gracias a un programa que tienen de puertas abiertas, en donde reciben visitas de estudiantes o personas interesadas en aprender sobre el proceso de elaboración de la cerveza. Aspecto que ayuda a las relaciones públicas ya que los visitantes salen optimistas del recorrido, ya que además de ser una experiencia única, se aprende.

Entre los aspectos que todos observamos fue una piscina en donde estaban reciclando las aguas residuales. Un programa de RSE que es velar por el medio ambiente.

Además de este tipo de actividades, esta empresa ha tenido otros reconocimientos:

Industrias La Constancia se hizo acreedora al premio regional por los procesos de elaboración de cerveza, en la categoría de materiales. Mientras que la mención especial se hizo en la categoría de agua por el proyecto Manejo sostenible del recurso hídrico, mediante la optimización del consumo de agua en el proceso productivo y la reducción de la carga orgánica contaminante presente en el afluente.

La empresa ha establecido programas de minimización de desperdicios y reciclado de materiales, también ha mejorado los precios de reciclado de películas plásticas y cartón en más del $300 \%$. También ha logrado reducir el $58 \%$ de los desechos sólidos enviados al relleno sanitario.

"El premio a la producción más limpia incentiva a la empresa a seguir siendo pionera en estándares de manufactura y de protección ambiental", recalcó Martin Van Dam, presidente de ILC.

El año pasado, ILC inauguró una planta de tratamiento de aguas residuales con una inversión de \$2.2 millones. La planta permite limpiar de residuos unos $1,040 \mathrm{~m}^{3}$ de agua por día, y permite aprovechar el proceso para obtener $1,000 \mathrm{~m}^{3}$ de gas metano e incluirlo en el proceso de generación de energía.

En varias ocasiones he realizado visitas con mis alumnos a Industrias La Constancia, en donde aplican diferentes modalidades de RSE, el objetivo es tener a todos los empleados motivados y además de realizar actividades que ayuden al medio ambiente. 
Siempre les comento a mis alumnos sobre un centro de cómputo que donó ILC, en donde los alumnos son niños que tienen a sus madres trabajando en "La Tiendona" y además algunos niños son hijos de mujeres que laboran en lugares de mala reputación como La Avenida y Zurita.

Además ha recibido premios por su aplicabilidad de la RSE, una empresa con liderazgo y catalogada como una de las mejores en El Salvador. Según la página Web de ILC, se constata su premio entregado por la Universidad Dr. José Matías Delgado en ranking de imagen. Los premios agenciados fueron Responsabilidad Social, Liderazgo y Reputación.

\section{La Responsabilidad Social Empresarial de Grupo Agrisal}

Eso que las empresas ayuda para evadir impuestos es un punto que no le quitaría el sueño a un empresario, ya que a través de la historia empresarial de esta empresa se preguntarán ¿A cuántas empresas y personas ha ayudado el grupo AGRISAL?.

Una estudiante de Diseño de la Universidad Dr. José Matías Delgado manifestó en un congreso de emprendedurismo de la Cámara de Comercio e Industria de El Salvador que su idea era trabajar para una empresa que fabricara ropa con añil. En ese emprendimiento tuvo que ver la Fundación Empresarial para la Acción Social FUNDEMAS. La ansiada alumna manifestó que Murray Meza le dijo "que quería que alguien exportara ese tipo de ropa a Japón". La alumna logró hacerlo y como el empresario le apadrinó, estaba agradecida. Sin embargo la soñadora siguió liderando su empresa gracias a la colaboración del filántropo empresario.

La RSE de este empresario ha dado frutos ya que los que han aplicado o han sido ganadores de becas trabajen en los hoteles de su propiedad o en otros lugares. La capacitación con estándares de calidad logran resultados óptimos.

Según Nelly Aparicio Gerente de Relaciones Corporativas de AGRISAL manifestó "se trata de un modelo especializado de trabajo en responsabilidad social". Las becas ganadas con el programa son una fortaleza en su estilo empresarial.

"Si perdemos de vista qué salvadoreño es el que queremos, si entendemos los valores de la civilización que podemos crear y los grandes costos que tiene apartarnos de esto, entonces vamos a estar bien, independiente de las ideologías; porque habrá suficiente gente con valores para no permitir que se hagan descalabros que nos perjudiquen a todos". 
Roberto Murray Meza, uno de los empresarios pioneros que le apostó a la Responsabilidad Social Empresarial. En el año 2001 nació Fundación Empresarial para las Acción Social FUNDEMAS, el objetivo fue contribuir al desarrollo económico y social sostenible de El Salvador. Esta asociación imparte capacitaciones y consultorías en el área de responsabilidad social y emprendedurismo relacionados a talleres EMPRETEC de las Naciones Unidas. Cuenta con 132 miembros y forma parte de organizaciones internacionales como Red Centroamericana de RSE (Integra RSE).

Entre las áreas de trabajo de FUNDEMAS están: RSE, comunidades, gobernabilidad, mercadeo responsable, proveedores, medio ambiente, política pública y público interno. Por lo tanto, son muchas actividades de RSE que ha realizado.

En el periódico salvadoreño El Mundo manifestaron que AGRISAL entregó becas a jóvenes, contemplado en el área de RSE. "Como representantes de la División Hotelera de Grupo AGRISAL, nos sentimos comprometidos con el desarrollo profesional de nuestra juventud. Estamos seguros que el compartir nuestra experiencia con estos jóvenes es la mejor forma de promover futuros líderes en la operación hotelera", expresó Carlos Mulhbach, director de operaciones de la División Hotelera de Grupo AGRISAL.

Un incentivo para un joven es ver coronada su carrera universitaria. Cuando los comensales y clientes visitaban los hoteles del Grupo AGRISAL, percibían el buen trato de becarios. De sueños que se plasmaban en capacitaciones, charlas, adiestramientos en cada habitación, en la cocina, en los pasillos y hasta en un cordial saludo en la entrada de los hoteles.

FUNDEMAS ayudó mucho al desarrollo empresarial salvadoreño, no importando si fuesen micro o pequeñas empresas.

FUNDEMAS trabaja con cuatro ejes EMPRESAL, EMPRETEC, CEFIEM Y FIDES.

Más de 20,000 alumnos de beneficiaron de los recursos tecnológicos patrocinados por el programas FUTUREKIDS en los centros juveniles Don Bosco.

¡EMPRENDE TU IDEA $i$ tiene por objetivo apoyar y potenciar a la micro y pequeña empresa en su desarrollo económico y sostenible a través de la Competencia e Planes de Negocios. Con este proyecto se crearon 16 empresas en el año 2003 de 150 planes de negocio.

Con el programa FUTUREKIDS se han beneficiado a 4,000 estudiantes para AGAPE en el Colegio san Francisco de Asís de Sonsonate. 
10,000 estudiantes participaron en el concurso CIBEROLIMPIADAS sobre tecnologías educativas.

Capacitaron a periodistas con el auspicio del TEC de Monterrey y Fundación Nuevo Periodismo.

Según FUNDEMAS, los beneficios que reciben las empresas al aplicar la RSE son:

Disminución de conflictos

Acceso a capital

Valorización de la imagen institucional y de su marca

Mayor lealtad del consumidor

Mayor capacidad de reclutar y mantener sus talentos

Sustentabilidad del negocio a largo plazo

Acceso a mercados

¿Cuál de los anteriores aspectos es el más importante? Los empleados de Almacenes Siman, Farmacias San Nicolás, el call center Atento, Industrias La Constancia, Hilasal. Super Selectos, etc., se muestran satisfechos ya que cuentan en sus empresas con todo lo indispensable para crecer y desarrollarse laborablemente.

Según el periódico La Página, en entrevista a Elena de Alfaro, quien expresó cómo FUNDEMAS mantiene la comunicación con las empresas en el contexto de la RSE y su misión en la ayuda de programa sociales:

¿Cómo FUNDEMAS mantienen una red de empresas?

Somos 126 miembros, entre los cuales tenemos fundaciones, empresas, personas particulares, instituciones, personal. Una buena parte son empresas en El Salvador. Dentro de esas 126 se tiene relaciones a nivel gremial. Por ejemplo CASALCO, la Cámara de Comercio que aglutinan mucho más. Realmente no son 126 sino que somos más de 4 mil personas que están siendo constantemente capacitados respecto al RSE.

Una empresa para poder empezar a poner en práctica el RSE es necesario inscribirse para capacitarse en FUNDEMAS?

No. Hay ciertos seminarios que son abiertos, para las pequeñas empresas y de hecho una de las cosas que me he propuesto es hacer que la RSE sea parte del cometido en una 
empresa, sin importar que la empresa sea pequeña, e invitar a esas personas a autoevaluarse para mejorar.

¿La labor de FUNDEMAS es difundir el RSE entre todas las empresas y hasta ahora lo han hecho a través de capacitaciones y seminarios?

Es correcto. Uno de mis principales objetivos cuando era parte de la Cámara de Comercio, justamente, fue difundir la RSE a los miembros. El $90 \%$ de los socios de la Cámara son pequeñas y medianas empresas.

FUNDEMAS ayuda mucho al factor empresarial salvadoreño, entre los logros alcanzados están:

Alianzas con 60 entidades de gobierno central, alcaldías, universidades, gremiales, ONG y empresas privadas.

Alianza con el Ministerio de Educación para incorporar el material educativo de LIMPIEMOS EL SALVADOR en la materia de Ciencia, salud y Medio Ambiente de los niveles de educación básica.

Se ha elaborado un material específico para las capacitaciones dirigida a los maestros denominado "Guía para el educador", el cual incluye veinte lecciones que enseñan de forma sencilla y dinámica, temas importantes sobre el medio ambiente y consejos para poner en práctica y formar parte del Programa LIMPIEMOS EL SALVADOR.

Se ha realizado más de 75 charlas de concientización, logrando impactar a 4,875 personas entre estudiantes universitarios, padres de familia y empleados.

Se ha realizado ocho jornadas de limpieza en diferentes puntos de El Salvador, contando con el apoyo de más de 3,510 voluntarios.

Las empresas necesitan que en conjunto gobierno, universidades y empresa privada trabajen en conjunto para desarrollar un país. Un país no puede desarrollarse si no empodera a los alumnos universitarios, no puede estar dividido con la empresa privada. En el ENADE 2013, Markt Kramer, disertó recientemente en un congreso en donde asisten empresarios, acerca que "Los problemas sociales, son oportunidades de negocio" bajo el concepto de "valor compartido" que acuñó junto a Michael Porter y que les supuso un premio Nobel.

Kramer exhortó: "que muchas veces los problemas sociales representan oportunidades de negocio para las grandes empresas ya que estas poseen los recursos que el gobierno no 
tiene; puntualmente citó ejemplos de: Coca Cola en las favelas de Brasil; Novartis en la India e ICl Lombard en Asia y los cultivos". Aclaró que el concepto valor compartido, no es filantropía y que es valor económico y beneficio social.

Es indiscutible saber que "el valor compartido" es un plus que las empresas pueden o deben tener para desarrollarse. Ayudar no solo implica hacer publicity o que la empresa tenga excelente imagen, todo está en los resultados, está en que los empleados y la comunidad sean beneficiadas.

\section{La Responsabilidad Social de las universidades salvadoreñas}

Actualmente con tanta problemática que embarga a El Salvador, es de suma importancia que se tome en cuenta temas como la responsabilidad social, en especial el que ejercen las universidades.

Según la Ley de Educación Superior (El Salvador), las universidades tienen tres ejes principales, impartir docencia, investigación y la proyección social, y es la proyección social la que permite a los centros de educación superior incorporar programas de responsabilidad social universitaria.

Las universidades como centros de educación superior tienen el deber de aplicar en sus diferentes cátedras la Responsabilidad Social Empresarial (RSE). Entiéndase la RSE como: "un imperativo estratégico que le permite a la empresa incorporar políticas y prácticas en beneficio de los accionistas, los colaboradores, la comunidad, el medio ambiente y toda su cadena de valor...". Tomado de Fundación Empresarial para la Acción Social (FUNDEMAS).

En el caso de las universidades, este tipo de estrategia empresarial, se puede incorporar en los departamentos o áreas de proyección social de las universidades, que es en donde más se puede ocupar la colaboración de los alumnos hacia las comunidades en la gestión y aplicación de proyectos. No confundir proyectos sociales con pintar árboles u otra actividad que no sea de beneficio a la sociedad.

Además, no confundir el término de responsabilidad social empresarial con filantropía; no tomar la RSE como una forma de donar. Las universidades cuentan con decanatos, personal administrativo, departamentos, catedráticos y alumnos, y es en donde se tiene que aplicar este tipo de programa. Las universidades tienen que contar con prestaciones hacia su personal, clínicas empresariales, guarderías, etc., con el objetivo de mantener motivado al personal y cumplir con la misión y visión empresarial. 
En un caso particular, la Universidad Francisco Gavidia, cuenta con una Dirección de Proyección Social, en donde involucra a los alumnos de las diferentes carreras a realizar proyectos que ayuden a las comunidades. Algunos de los proyectos que han realizado son: deshidratación de frutas, talleres para la elaboración de artesanías, proyectos agrícolas y técnicos, capacitaciones en el área turística, empresarial, etc. Además, se implementó desde el año 2003 un programa humanitario denominado "UFG AYUDA", el cual soy uno de los fundadores. El principal objetivo del programa es de llevar víveres, ropa, piñatas, sillas de ruedas, etc. a personas de escasos recursos en todo el país.

Actualmente la UFG está participando en programas de conservación de tortugas marinas en colaboración con La Fundación Zoológica de El Salvador FUNZEL en Isla Méndez en la bahía de Jiquilisco, Usulután.

Cuando aplicamos programas de ayuda a las comunidades pobres y se involucran a los estudiantes, se les sensibiliza; aprenden a ayudar y a realizar actividades en bien de la sociedad. Esto en el futuro les ayudará a ser unos ciudadanos de bien y lo aplicarán en las empresas que laboren o creen. Cada universidad tiene que planificar qué actividades se pueden realizar, teniendo en cuenta la zona geográfica, aspectos financieros, personal, y acordes a las necesidades de cada población.

Lo recomendable para que se aplique una gestión óptima de la RSE sería que el gobierno, la empresa privada y oenegés unieran esfuerzos para cumplir los propósitos planificados por las universidades. Sin embargo, hay casos en los que ya se han involucrado para trabajar en conjunto.

Las universidades no pueden estar solamente enseñando como una pedagogía bancaria, en donde sólo se les inculca a los alumnos a ser empleados y a crear empresas, se les debe enseñar también sobre la realidad del país e incorporar proyectos que ayuden al desarrollo económico y social. Al final, se logra las metas en áreas técnicas, culturales y artísticas, generación de empleo (en especial a jóvenes), etc.

Por lo tanto, las universidades tienen que establecer en su planificación anual programas de responsabilidad social universitario para el bien tanto de los empleados, alumnos y la comunidad en general. Actividad que ayuda a difundir valores, una filosofía empresarial con énfasis en el desarrollo humano. 


\section{Conclusión}

La responsabilidad social empresarial es una estrategia que ayuda a crear imagen, motivación entre los empleados y una cultura organizacional ejemplarizante de gestión empresarial. La RSE busca que todas las áreas de la empresa se integren y formen un sistema con valores que contribuyan al desarrollo tanto de la empresa como sus públicos. Los ejemplos expuestos demuestran que en El Salvador existen empresas que se comprometen con los empleados y sociedad en buscar soluciones óptimas para el buen funcionamiento de la planificación en el ámbito de RSE.

Si en las organizaciones los jefes o gerentes la aplican, tendrán mejores resultados, ya que los empleados tendrán satisfacciones personales, las cuales concluirán con el objetivo de tener a empleados motivados y satisfechos en el mundo laboral.

Las universidades, deben de incentivar a todos los alumnos, catedráticos, personal a que incorpore programas de RSE. Sabemos que un país vive en mejores condiciones si se aplican verdaderos programas de RSE, desde la planificación de una fiesta infantil con niños de escasos recursos hasta ludotecas y programas de alfabetismo. Cada carrera cuenta con las fortalezas necesarias para aplicar la RSE hacia la sociedad. Al final, los aplicadores verán los resultados al ayudar a la sociedad.

Un mundo mejor será cuando los seres humanos actuemos responsablemente en la conservación del medio ambiente, en colaborar con la comunidad, en participar con el Gobierno y empresa privada en programas de responsabilidad social empresarial.

Las mejores organizaciones obtienen resultados óptimos en los públicos a los que lleva su actividad empresarial, ya sean público interno y público externo. Los casos expuestos son una parte nada más hasta donde pueden llegar las empresas al aplicar planes de RSE.

\section{Bibliografía}

ENADE XIII, Encuentro Nacional de la Empresa Privada, Mejorando empresas, transformando vidas, 2013.

Martínez Martínez, Karla Liliana y Sorto Alvarenga, Sonia Gabriela, Tesis "La Responsabilidad Social de los Medios Televisivos y Radiofónicos en la Emisión de Campañas de Acción Social. 
R Wilcox L. Dennis, Relaciones Públicas estrategias y tácticas „¿qué son las relaciones públicas?.

\section{Consultas virtuales}

- http://www.fundemas.org/index.php?option=com_content\&view=article\&id=23\&ltemid= 39

- http://www.laprensagrafica.com/economia/nacional/174338-la-rse-es-rentable-paralos-negocios.html

- http://archive.laprensa.com.sv/programas/impresion/imprimir.asp?EIUrl=http://archive.I aprensa.com.sv/20060704/eleconomista/529502.asp

- http://www.laprensagrafica.com/economia/nacional/27504-premian-a-la-constanciapor-su-produccion-limpia.html http://www.plazatempo.com/announcements/rse-agrisaluna-vision-integral

- http://www.elsalvador.com/mwedh/nota/nota_completa.asp?idCat=6374\&idArt=482152 7

- http://elmundo.com.sv/grupo-agrisal-presento-a-jovenes-becarios

- http://www.lapagina.com.sv/entrevistas/33707/La--Responsabilidad-SocialEmpresarial-da-una-mejor-herramienta-para-ser-mas-competitivo 9 de junio del 2010

- http://www.elimportuno.com/fiesta/item/6890-la-responsabilidad-social-de-lasuniversidades-salvadore\%C3\%B1as

- http://www.vanguardia.com/historico/91451-la-responsabilidad-social-empresarial-noes-solo-para-grandes-empresas

- http://www.laconstancia.com/?art=1071\&title=RECONOCIMIENTOS\%20PARA\%20ILC \%20EN\%20EL\%20RANKING\%20DE\%20IMAGEN\&lang=es

- https://www.youtube.com/watch?v=H-LPtPrc_q8

- http://rse.camacoes.com.sv/pages/viewnew.asp?CodArt=199

\section{Forma de citar este artículo en bibliografías}

LÓPEZ, F. A. (2014): La Responsabilidad Social Empresarial: aplicándola para tener un mundo mejor, en Revista PANGEA, 5, páginas 74 a 89. Red Académica lberoamericana de Comunicación. Recuperado el _ de _ de 2 _ de: http://www.revistapangea.org 